\title{
Deployment of the Festo PA Workstation for Undergraduate Training on Industrial Process Automation
}

\author{
Patrick Onotu ${ }^{1}$, Uche M. Dimkpagu ${ }^{2}$ \\ ${ }^{I}$ Department of Electrical/Electronic EngineeringTechnology Akanu Ibiam Federal Polytechnic, Unwana, \\ Ebonyi State, Nigeria \\ ${ }^{2}$ Department of Electrical/Electronic EngineeringTechnology Akanu Ibiam Federal Polytechnic, Unwana, \\ Ebonyi State, Nigeria
}

\begin{abstract}
Industrial automation involves the use of machines, control systems and information technologies in optimizing productivity in the production of goods and delivery of services. The Festo compact process automation (PA) workstation is a piece of laboratory equipment designed for the training of process automation engineers. It consist of programmable logic controller (PLC) rack, output devices (including several valves, a motor, a centrifugal pump etc), input devices (including flow sensor, heat sensor, level sensor, pressure sensor), switches, network of pipes, two storage tanks a heating and a cooling system. This paper presents the automation of liquid control process implemented on the PA workstation using PLC programming, manual liquid process control using Human Machine Interface (HMI) and Supervisory Control and Data Acquisition (SCADA) system. These devices and systems are all networked together with the workstation through Ethernet and Field-Bus (Profibus) technology. Process visualization from HMI and SCADA runtime screens are presented and analyzed to validate the integrity of the PA workstation in implementing process control. The results obtained shows that the workstation can mimic most industrial processes and deployable for the enhancement of students' training on process automation.
\end{abstract}

Keywords: Industrial automation, Festo compact workstation, Programmable Logic Controllers, HMI and SCADA systems.

Date of Submission: 05 November 2016

Date of Accepted: 18 November 2016

\section{INTRODUCTION}

Automation can simply be defined as the use of machines, control systems and information technologies in the production of goods and delivery of services. An industrial automation system, also known as Process Automation System (PAS) is used to automatically control most industrial processes. From the analysis presentedin [1], an industrial process could involve the following:

a) Change of energy state, such as from hot to cold, or liquid to gas and distillation of water;

b) Change of composition, as occurs in a chemical reaction or in mixing;

c) Change of dimensions, as in breaking of coal;

d) Change in level, as in reservoir or tank filling;

e) Change in media velocity, as in a reservoir feed pipe,

f) Maintaining a pressure level, as in a water distribution network.

The above processes are as seen in chemical industries, oil refineries, paper and pulp factories etc. The PAS often uses a network of various interconnect sensors, controllers, operator terminals and actuators in conjunction with computer technology and software engineering and it is associated with Supervisory Control and Data Acquisition (SCADA) systems, a technology used to remotely control processes.

In this paper, automating a liquid control process with the use of PLC, HMI, SCADA system and FieldBus technologies in a compact process automation workstation is demonstrated. The task involves heating, cooling and circulation of water through the workstation, the indication of critical water levels, temperature and flow rate in a closed-loop control system. The task is analogous to industrial process automation especially in water processing industries and helps visualize the big picture of automating a process.

The task is divided into three stages namely:

a) Automatic process control using step 7 PLC programming software in ladder;

b) Manual process control and visualization with HMI screens; and

c) Manual process control and visualization with SCADA system and Field-bus technology. 
The paper is structured such that the background information on PLC, HMI, SCADA and Field-Bus are detailed in section II. The methods used in implementing this project and the equipments are detailed in section III. The PLC program, the HMI screens and the SCADA system designs and implementations with Screen shots are presented in section IV while the result from the implementation are discussed in section V.

The introduction of the paper should explain the nature of the problem, previous work, purpose, and the contribution of the paper. The contents of each section may be provided to understand easily about the paper.

\subsection{Programmable Logic Controller}

\section{II.PROCESS AUTOMATION DEVICES AND SYSTEMS}

A programmable logic controller (PLC) is a special form of microprocessor-based controller that uses programmable memory to store instructions and to implement functions such as logic, sequencing, timing, counting, and arithmetic in order to control machines and processes [2].

Input devices in the form of sensors and switches and output devices in the form of motors and valves in the system to be controlled are connected to the PLC. The PLC is then supplied a sequence of instructions which is stored in its memory. The controller performs its function by monitoring the inputs and outputs according to these instructions or program. A PLC is very flexible in usage in that to perform a different control task, another set of instructions are downloaded into its memory and no need of any rewiring or reconfigurations. Therefore, the same controller can be used to implement a wide range of control tasks and this is an advantage over other types of controllers [3]. The structure of a PLC system is shown in figure 1.

\subsubsection{Merits and Demerits of PLC}

As seen in [4] some of the advantages of PLCs are as follows:

a) Optimised for industrial application to withstand vibrations, temperature, humidity and noise;

b) Easily expanded with more inputs/outputs, controllers and other cards;

c) Software based therefore easy to reprogram and to add functionality without rewiring;

d) Controllable and Reliable due to showing fault codes when there is a hardware or software problem;

e) One Controller Box, instead of a lot of components

f) Takes up a lot less space since the PLCs are made more compact;

g) Cost effective for medium to large systems;

h) Repeatable 24/7 operation;

i) Faster due to quicker transistor switching time compared to an inductive relay.

The following are the disadvantages:

a) Cost ineffective on a very small scale (although very small PLCs are available)

b) Cannot handle large amounts of data like (video, sound files etc.)

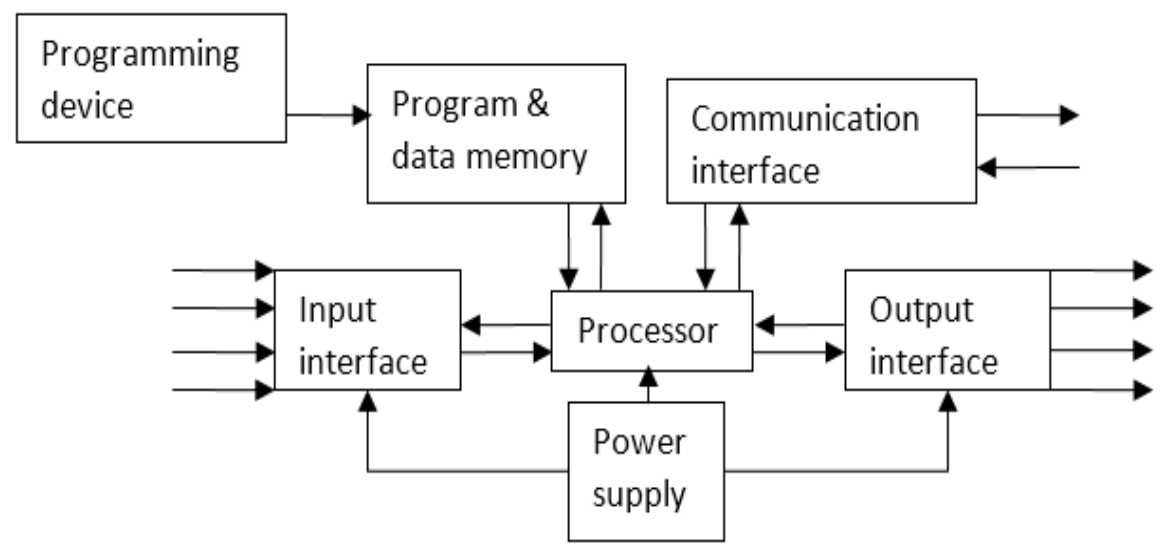

Fig.1. PLC hardware system

\subsection{Human Machine Interface}

A Human Machine Interface (HMI) is a system that provides the interface between a system operator and a process. Though the PLC is the actual unit which controls this process there is an interface between the operator and the HMI running on WinCC flexible and an interface between WinCC flexible and the PLC. The HMI system performs the following tasks: 
a) Provides process visualization;

b) Allows operator control of the process;

c) Display alarms and messages;

d) Archiving process values and alarms etc.

\subsection{Supervisory Control and Data Acquisition System}

Supervisory Control and Data Acquisition System (SCADA) is a real time industrial process control system used to centrally monitor and control remote or local industrial equipment such as motors, valves, pumps, relays, sensors, etc. SCADA has a wide range of application in controlling chemical plant processes, oil and gas pipelines, electrical generation and transmission equipment, manufacturing facilities, water purification and distribution infrastructure etc. It is a system consisting of a number of remote terminal units (RTUs) collecting field data connected back to a master station via a communication system. The structure of a SCADA system is shown in figure 2 below.

\subsubsection{Benefits of SCADA}

a) Improved operation of the system;

b) Increased productivity of the personnel;

c) Improved safety of the system due to better information and improved control;

d) Protection of the plant equipment;

e) Safeguarding the environment from a failure of the system;

f) Improved energy savings due to optimization of the plant;

g) Improved and quicker receipt of data so that clients can be invoiced more quickly and accurately [4].

\subsection{Networking of the Automation System}

The schematic diagram of an automation system involving SCADA system, HMI and PLC with Profibus and Ethernet communication media is shown below. This illustrates how the devices and systems are networked to local and remote process control.

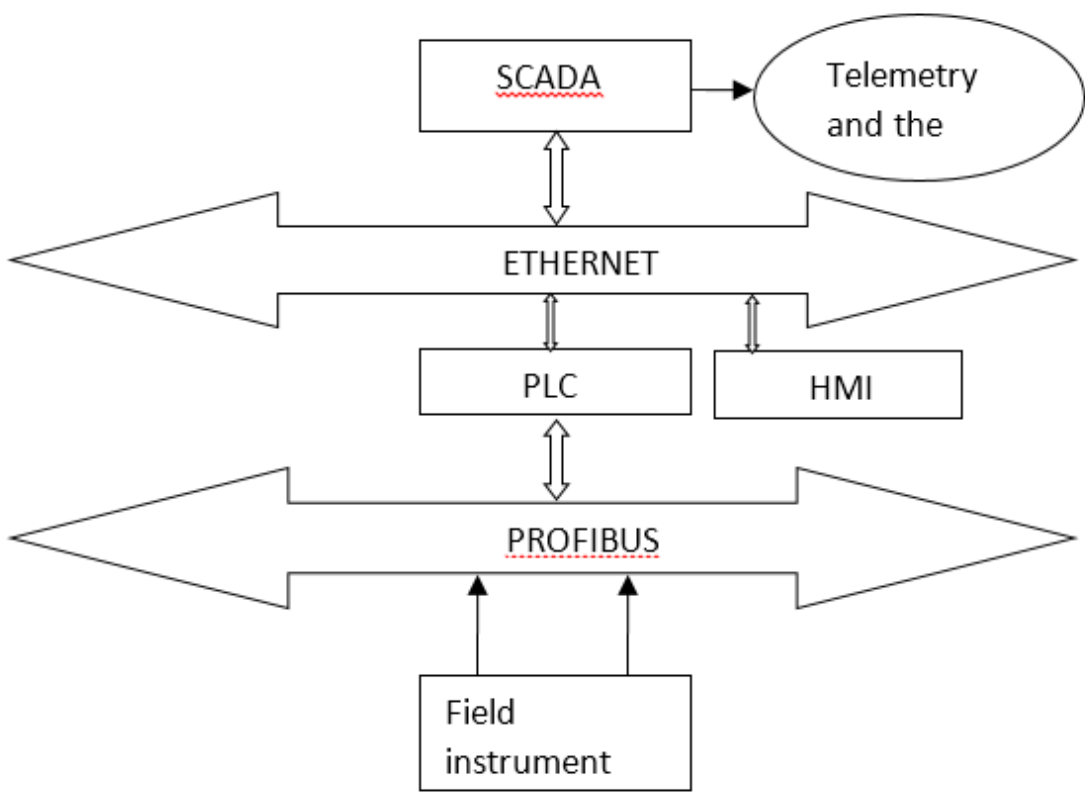

Fig. 2.Schematic diagram of an automation system

\section{MATERIALS AND METHODS}

Figure 3 below shows an overview of the components and structure of the Festo compact process automation (PA) workstation as a network of sensors, actuators, pump, valves, water tanks, heater, cooler and PLC rack [5]. 


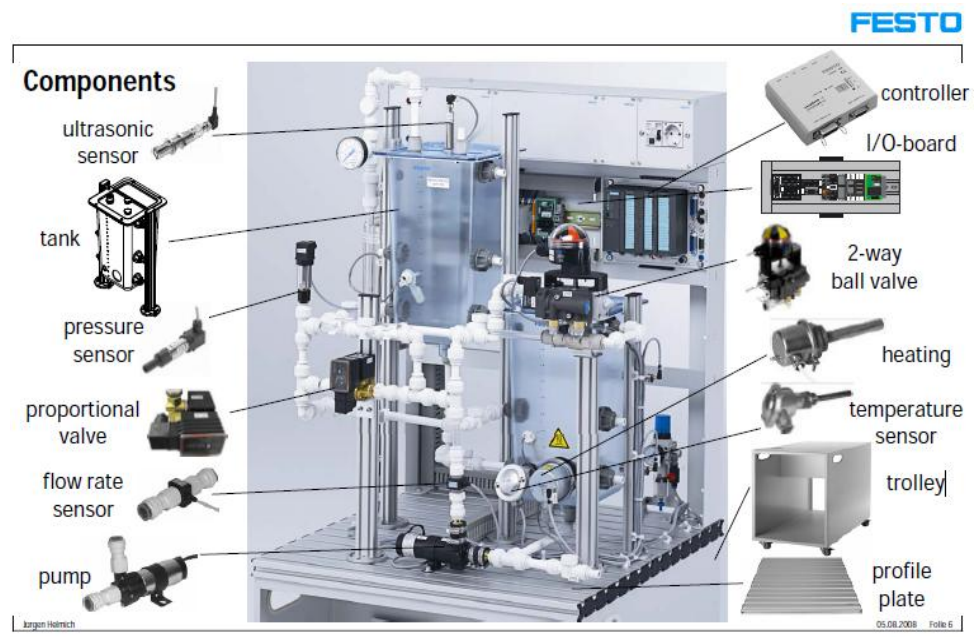

Fig. 3. Festo PA workstation

(Source: Festo Module Commission Documentation)

The PLC installed on the workstation is the Simatic S7 300 with 314-6EH04OABO central processing unit (CPU). The HMI device also mounted on the platform is the six inch Simatic Touch Panel TP170PN/DP. A SCADA system is connected to the station via Automation System, Simatic PCS7 AS RTX with a soft PLC and an external I/O unit, Simatic ET 200M/Link [5]. The above workstation is designed such that the following can be performed on the process elements:

a) Heating of water with the electric heater located in the lower tank;

b) Cooling of water with the cooler located below the profile plate;

c) Pumping of water from the lower tank into the upper tank and back through the pneumatic 2-way ball valve;

d) Water level measurement using the ultrasonic, capacitive and float level sensors;

e) Water temperature measurement using the temperature sensor;

f) Water flow measurement using the flow sensor;

g) Water pressure measurement using the pressure sensor;

h) Implementation of automatic and manual process using PLC, HMI, SCADA and Field-Bus technology.

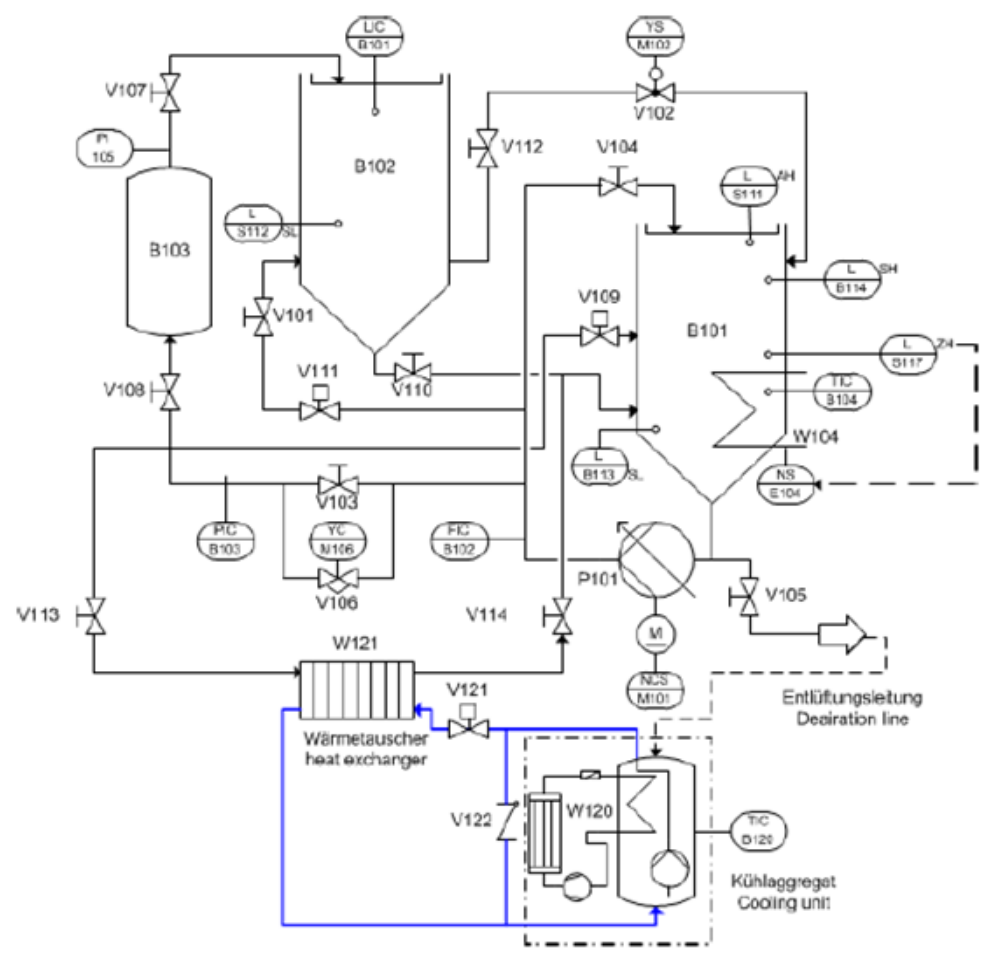

Fig. 4. P \& ID of Festo PA workstation 


\section{IMPLEMENTATION OF THE PROCESS AUTOMATION}

4.1 PLC Implementation

The following flowchart shows the algorithm for automating the process control using PLC.

Heating, Cooling and Circulation of Water

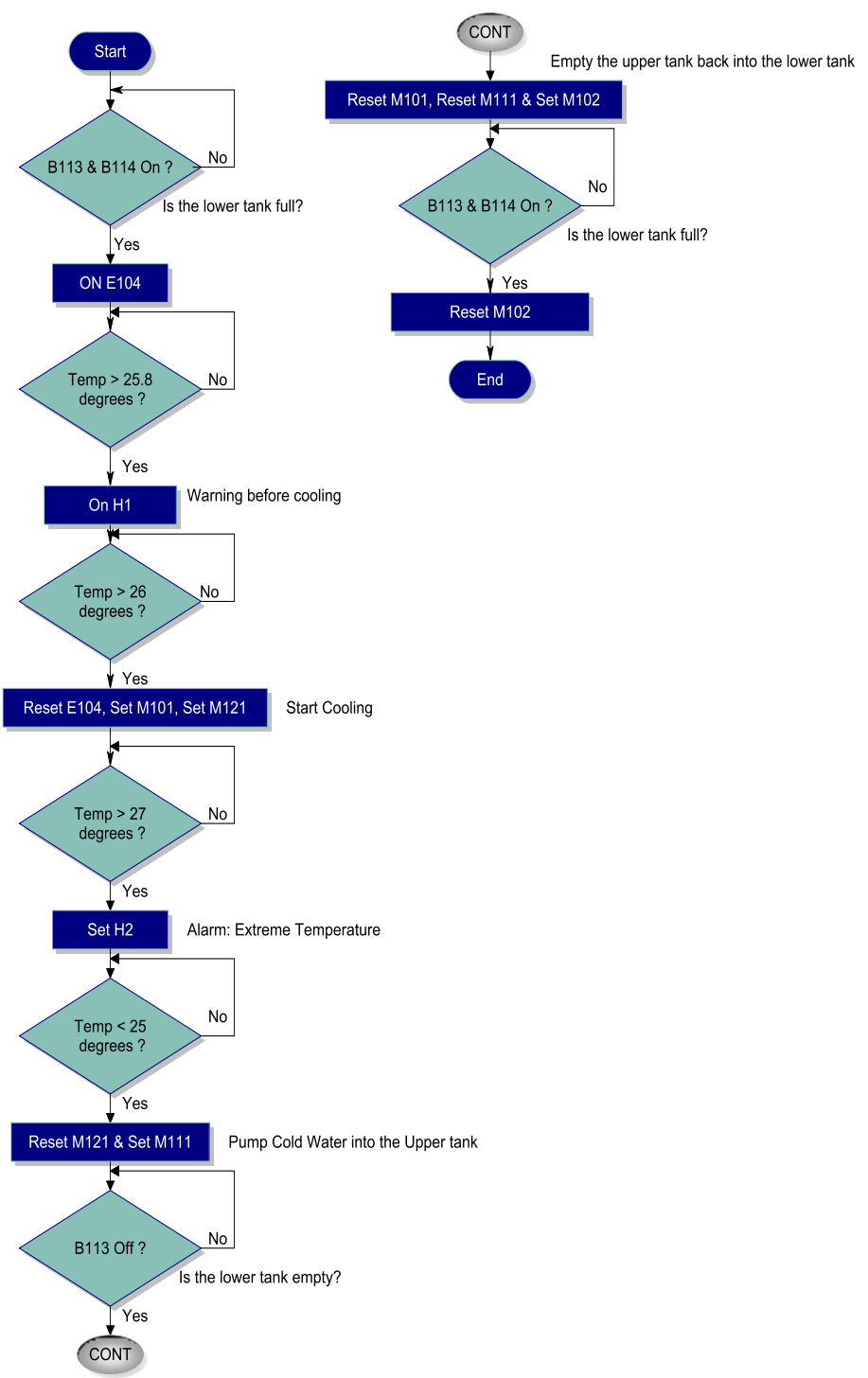

Fig. 5 Process automation flowchart

This algorithm was implemented using ladder programming language and the results are discussed below in result discussion section. The said ladder program cannot be presented here due to its volume.

\subsection{HMI Implementation}

Four HMI Screens, shown in figure 6, were designed to manually control and visualise the process namely:

The Main_Screen which carries the label and task of this project and a link to the other three screens;

The Process screen on which the pictorial implemention of the entire process is displayed;

Data_Trending screen on which the variation of temperature with respect to time is displayed graphically; and Alarms\&Messages screen on which an alarm or warning message is displayed for any critical water temperature. The other three screens can be switched to and back from the Main_Screen.

The events of different switches were set to switch on the M101, M102, M121, M111 and E104. Switches with graphics were used to match a switch with its corresponding output device as shown on the process screen of figure 6(b) for easy identification. Three I/O fields were tagged with analogue inputs AI_Flow, AI_Level and AI_Temp parameter scaling functions to display the real-time flow rate, upper tank level and temperature respectively [6]. 


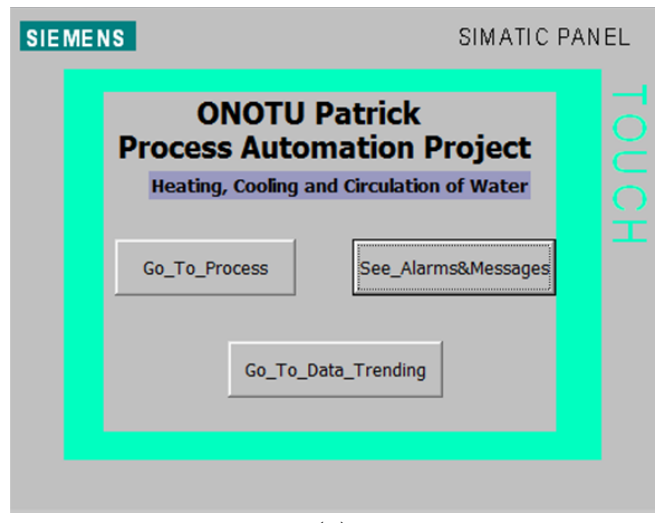

(a)

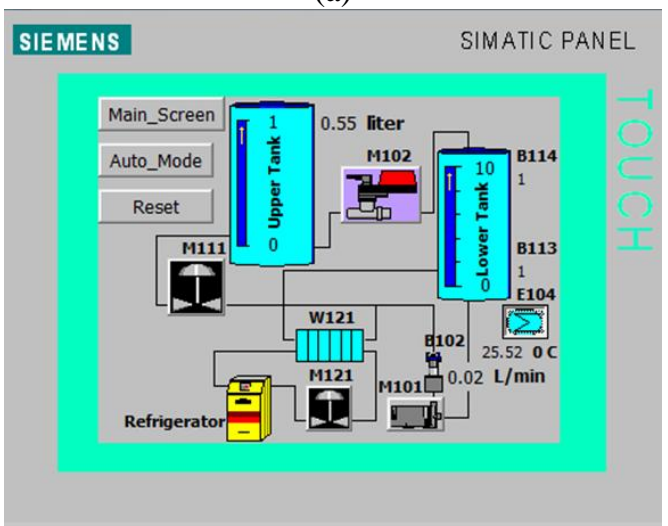

(b)

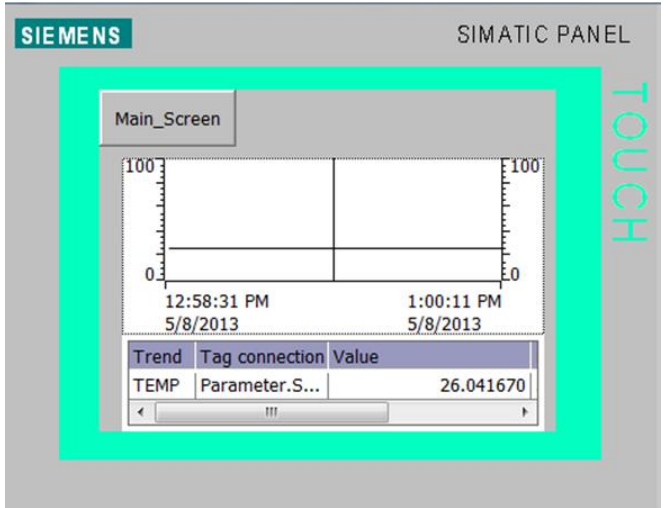

(c)

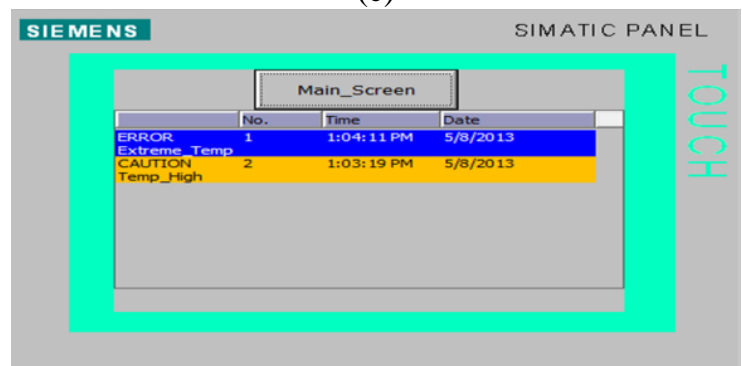

(d)

Fig. 6. (a) Main_Screen (b) Process (c) Data_Trending (d) Alarms\&Messages

\subsection{Implementation with PCS7 SCADA System}

The Simatic process control system (PCS 7) is a software package used in conjunction with WinCC graphic designer installed in theEngineering System (ES) to implement a SCADA operation [7]. The ES is connected to an Automation System (AS) using an Ethernet cable and the AS is connected to the plant using profibus. System 
network configuration is carried out to enable data communication. In this project an AS with a virtual PLC is used in conjunction with the Festo compact PA workstation as the process plant. The SCADA picture designed for the above process is presented in figure 7 below.

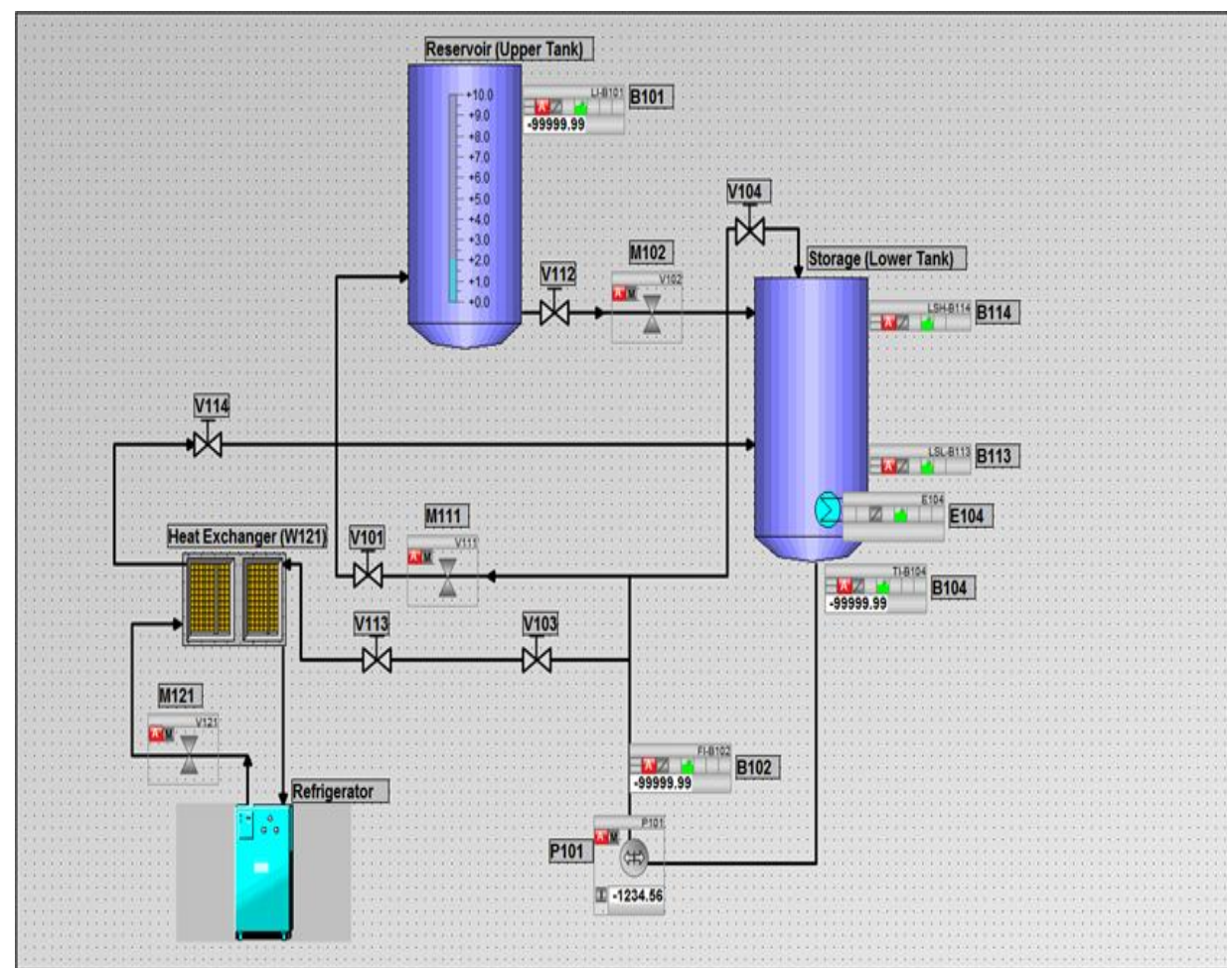

(a)

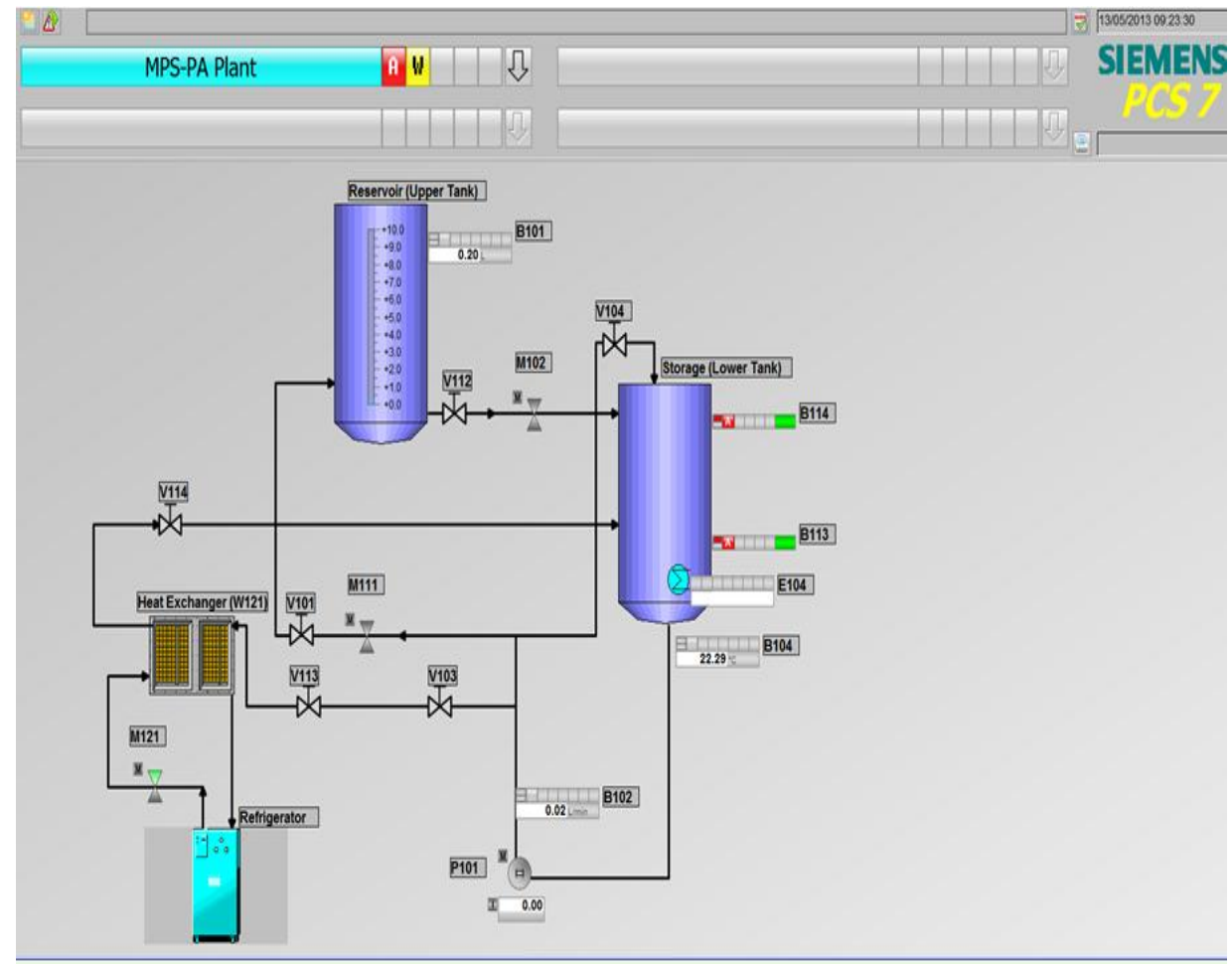

(b) 


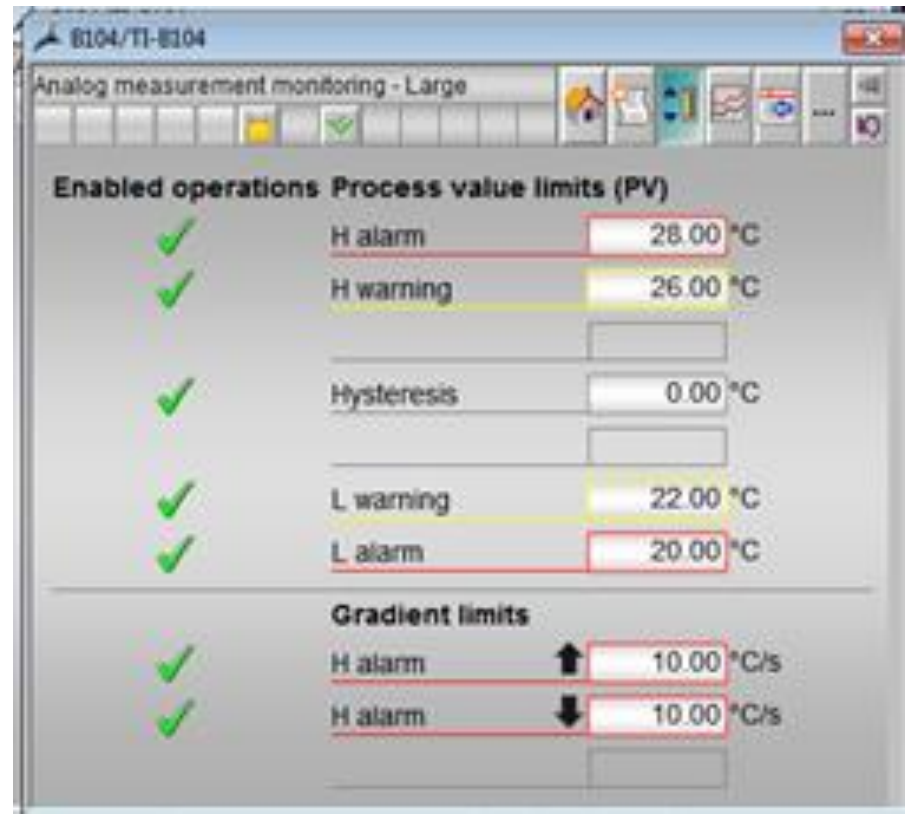

(c)

Fig. 7. SCADA screen shot of (a) the pictorial design (b) runtime before process start and (c) alarm and warning settings

As shown in figure 7(b), the storage tank is filled and heating can start. Temperature upper and lower limit alarms are set at $28^{\circ} \mathrm{C}$ and $20^{\circ} \mathrm{C}$ respectively from figure $7(\mathrm{c})$.

\subsection{PLC Implementation}

\section{RESULT DISCUSSION}

The program for the Siemens PLC was written using step 7 ladder language. The program was downloaded into the PLC and used to implement the liquid heating and cooling control. The results of the implementation showed that process automation can be effectively demonstrated with Festo PA workstation to a high control precision. It is impossible to present the ladder language here due to its volume but available as an appendix.

\subsection{HMI Control}

HMI screens were designed as shown in figure 6 and used to run the process specified in section IV above manually by switching on/off E104, M111, M121 and M102 directly and visually determining when to start/stop heating, cooling and circulation. For automatic operation, the program in section 4 was written in ladder in an operating station running Siemens Step7 programming software and downloaded to the PLC after its configuration. The activities of the PLC were displayed on the HMI screens designed for specific tasks. The following are the displayed activities:

Stage 1 - The start button was pressed when the heating condition was satisfied and heating started. When the temperature was greater than $26^{\circ} \mathrm{C}$, valve 121 opened, M101 switched on and cooling started automatically as programmed. The Process screen shows the increase in flow rate to confirm water pumping through the cooling circuit as there is no increase in the upper tank level. Temperature $=26.74^{\circ} \mathrm{C}$ and flow rate $=3.471$ itre $/ \mathrm{min}$

Stage 2 - As the temperature dropped below $25^{\circ} \mathrm{C}$, cooling circuit was closed while valve 111 opened to allow cold water into the upper tank automatically. The upper tank level began increasing. The flow rate also increased as more water is allowed through the new channel.

Stage 3 - There was an increase in the temperature from $25.5^{\circ} \mathrm{C}$ to $27.3^{\circ} \mathrm{C}$ with respect to time. A sudden increase in temperature was observed as soon as cooling started and triggered the alarm. This was shown in the signal trend.

Stage 4 - The proximity sensors B113 and B114 were in the off states meaning that the cold water has been pumped into the upper tank. The lower tank level reduced to minimum while the upper tank has risen to 8.45litres as programmed.

Stage 5 - The pneumatic valve M102 opened when B113 was off to empty the upper tank back into the lower tank. The upper tank level now at 2.13 litres while the lower tank proximity sensor B114 was back on. 


\subsection{Alarms and Messages}

A sudden increase in temperature was observed at the point of opening of the cooling circuit, the reset indicator light powered on as programmed. The start button indicator light was powered on and a warning message displayed on the HMI Alarms\&Messages screen when the temperature of the water was greater than $25.8^{\circ} \mathrm{C}$. This alerted the operator that the set point of the water temperature is almost reached and necessary actions should be taken. No action was necessary in response to the alarm because the heating already stopped before its trigger.

\subsection{Stop/Reset Buttons and the Auto_Mode Switch}

The stop button was programmed for process termination in an emergency. The button was pressed during the cooling of water and M101 and M121 were switched off, with no regards to the conditions of the comparators, in confirmation of its function. The reset button was used to continue the process by resetting M150.0 and also the alarm. The Auto_mode switch on the HMI Process screen is tagged with the start button and performs same function.

\subsection{SCADA Control}

In figure 8 (a) E104 was switched on (indicated by its green colour) and the water heated to $27.05^{\circ} \mathrm{C}$, the intended upper limit was $27^{\circ} \mathrm{C}$. The storage tank was filled before heating (B113 and B114 were green indicating their on state). A warning message was flagged as the water temperature was above $26^{\circ} \mathrm{C}$ as indicated by the yellow colour on the alarm bar. A uniform rise in temperature with time was displayed using the designed temperature trend on the bottom right hand side of the screen.

In figure 8 (b) a sudden rise in temperature, above $29^{\circ} \mathrm{C}$, was observed as the cooling circuit was opened, this triggered an extreme temperature alarm shown by the red colour on the alarm bar. E104 turned white indicating it's off state while pump P101 turned green indicating it's on state. M121 was on (green) showing circulation of cold water.

The water was cooled as shown in figure 8 (a) below the lower set point of $25^{\circ} \mathrm{C}$ and the cold water pumped into the upper tank through M111 with V103 closed and V101 opened until B113 and B114 turned off. P101was then switched off with flow rate approximately zero indicated by the white colour of B102.

The temperature trending shows a uniform decrease until M111 was opened at $24.92^{\circ} \mathrm{C}$. This triggered a disturbance in the temperature fall as shown in figure 8 (a). In figure 8 (b) M102 and V112 were then opened to empty the upper tank into the lower tank. As the water level passes B113 and B114 alarm was triggered calling for the close of M102. The alarm was acknowledged thereafter.As the water flows into the storage tank, due to the temperature difference between water in the two tanks, a sharp drop in temperature from $24.5^{\circ} \mathrm{C}$ and $24.05^{\circ} \mathrm{C}$ was observed and it reflected in the trend of figure 8 (b). Further drop to $23.73^{\circ} \mathrm{C}$ was observed when the pump was switched off. The water temperature remained approximately at this value until the end of the process. The lower limit alarm was never triggered.

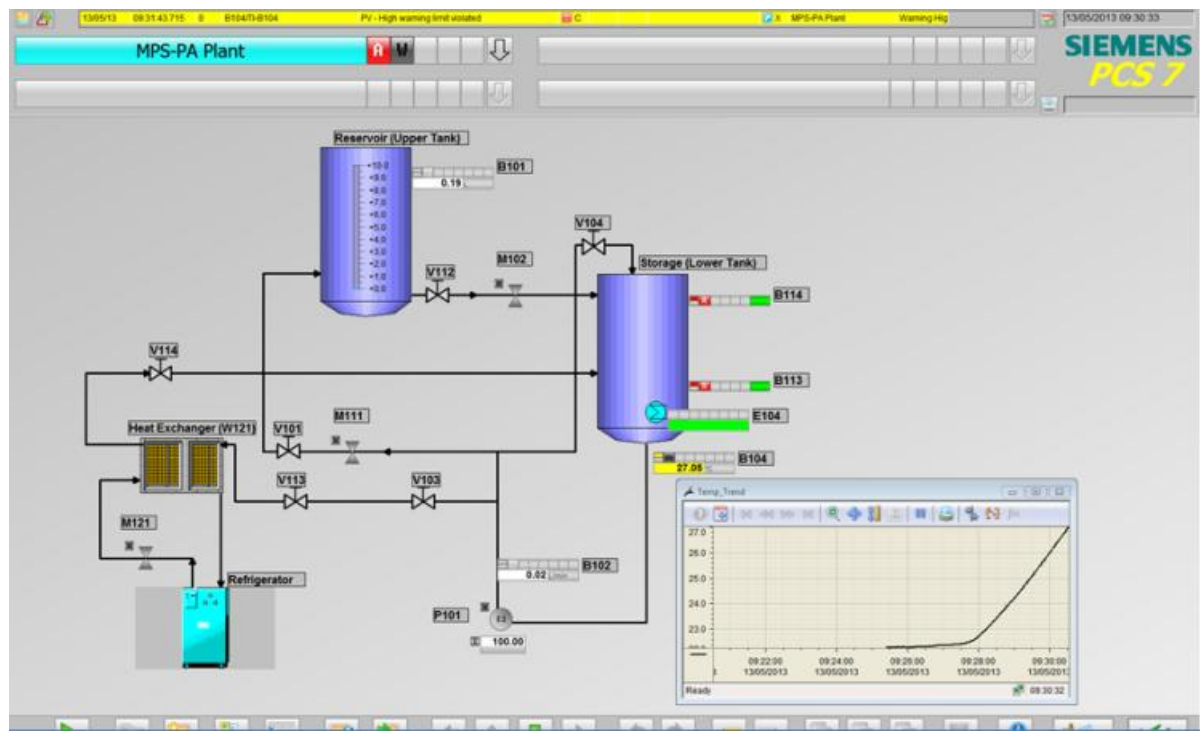

(a) 


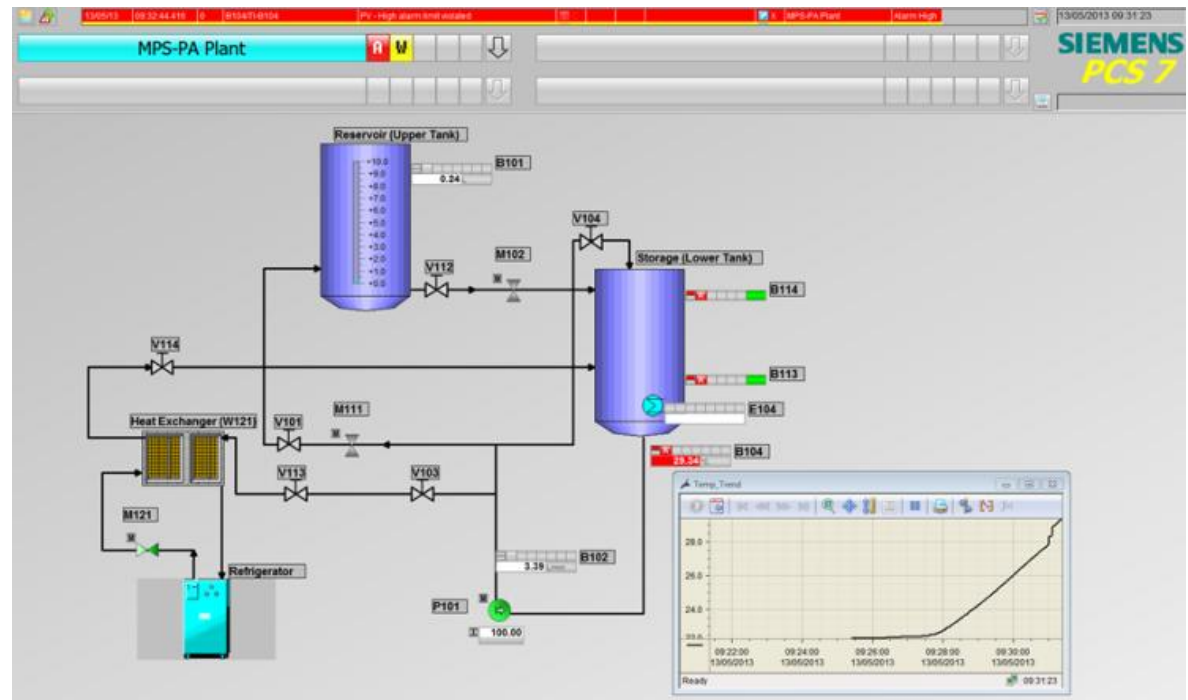

(b)

Fig. 8. SCADA screen shots of (a) heating start, warning and temperature trending (b) cooling start, alarm and temperature trending

\section{PERFORMANCE EVALUATION AND CONCLUSION}

A liquid control process has been implemented with PLC, HMI, SCADA/Field-Bus technologies. The process control was automated using Simatic step 7 PLC programming in ladder and manually implemented with HMI device and SCADA system. The automated process control with PLC produced the best control results as set points of water temperature and level for the opening and closing control valves and starting and stopping of the pump were all accurately adhered to. In the manual process control with HMI device there was always a time lag between a set point and the required corresponding actions due to human error. These delays were more pronounced with SCADA system because of the components switching procedure and could be unfavorable in production and manufacturing control system applications were attention is paid to precision. These delays are evident in figure 7 (a) and (b), before the cooling circuit was opened the temperature had risen to $27.95^{\circ} \mathrm{C}$ where the upper limit was $27^{\circ} \mathrm{C}$.

It is worthy of note, therefore, that for accuracy of process control specifications and improved productivity level, automatic operations should be employed in process industries where possible. These will help check human errors and improve plant safety and availability. As were observed in the automated part of this project, there were no time delays between starting of the pump and opening valves and pumping water from the storage tank after the lower level sensor turned off.

In conclusion, the possible liquid process control with the available devices on the Festo platform have been implemented and results discussed in details. It has been clearly shown that various automatic control activities can be demonstrated in the laboratory making undergraduate training a lot easier. The deployment of this workstation can help improve the practical experiences of automation and control students prior to graduation.

\section{REFERENCES}

[1]. Hongwei Z. (2012). Introduction to Industrial Automation and Process Control. Faculty of Arts Computing, Engineering and Sciences. England. Sheffield Hallam University.

[2]. Bolton W. (2009). Programmable Logic Controllers. [Electronic Book]. 5th Edition. Oxford. Elsevier Ltd. Book from Engineering Village last accessed 1st May, 2013 at: http://wobl.engineeringvillage.com/

[3]. Wesseler M. (2012). PLC programming. Faculty of Arts, Computing, Engineering and Sciences. England. Sheffield Hallam University

[4]. Hongwei Z. (2012). SCADA, Field-bus and DCS. Faculty of Arts, Computing, Engineering and Sciences. England. Sheffield Hallam University.

[5]. Festo (2001). Collection of Data Sheets. Festo Didactic GmbH \& Co.

[6]. Festo (2012). Cooling Unit with Heat Exchanger. [Online]. Last accessed 1st May, 2013 at:

[7]. http://www.festo-didactic.com/int-en/learning-systems/process-automation/accessories/cooling-unit-with-heat-exchanger/

[8]. Siemens (2008). Simatic HMI WinCC User Manual. 